\title{
Personalised Spam Filter for Social Networks Using Machine Learning Algorithms
}

\author{
Mohammed Husain ${ }^{1 *}$, Antarang Poogalia ${ }^{2}$, Devan Somani ${ }^{3}$, Gauri Walke ${ }^{4}$, Akash Agrawal ${ }^{5}$, \\ and A. J. Agrawal ${ }^{6}$ \\ 1,2,3,4,5,6 Shri Ramdeobaba College of Engineering and Management, Nagpur, India
}

\begin{abstract}
Social Network is one of the well-known and reliable mediums for communication which has made the whole of the world 'A Global Village' and where people easily connect with each other. With this boom of internet, many instant social media platforms have brought exploration of interactivity among users. With this rapid evolution, users of social networking sites may easily get overwhelmed by the excessive volume of information feeds and may also face difficulties to find truly valuable information. This paper introduces a personalised feed system for users based on their interests and displaying the content accordingly. In this paper the different machine learning algorithm with natural language processing to classify messages were used which leads to a time saving and user-friendly system.
\end{abstract}

KEY WORDS: SOCIAL NETWORKS, PERSONALISED FEED SYSTEM, NATURAL LANGUAGE PROCESSING, MACHINE LEARNING.

\section{INTRODUCTION}

An online platform on which people connect with same interest is basically referred as social network. These platforms provide a medium to connect with individuals of similar or different interests creating a hub. Since a huge hub participates on these platforms, the user can receive a high volume of messages from different individuals creating a chaos and unwanted messages. These messages sometimes contain a true information and sometimes false, which leads to a state of confusion in the minds of the users and leads to first step towards spam messaging.

Spam messages means an irrelevant and unsolicited message sent by an known/unknown user which may

\section{ARTICLE INFORMATION}

*Corresponding Author: husainmq@rknec.edu

Received 19th Oct 2020 Accepted after revision 23rd Dec 2020

Print ISSN: 0974-6455 Online ISSN: 2321-4007 CODEN: BBRCBA

Thomson Reuters ISI Web of Science Clarivate Analytics USA and Crossref Indexed Journal

\section{1) Clarivate}

NAAS Journal Score 2020 (4.31)

A Society of Science and Nature Publication,

Bhopal India 2020. All rights reserved.

Online Contents Available at: http//www.bbrc.in/

Doi: http://dx.doi.org/10.21786/bbrc/13.14/87 lead to a sense of insecurity among users. These messages affect user's emotions and privacy and hence affect the lives of individuals. To deal with this problem there are a numerous spam detecting and filtering applications available. Most of these applications detect the spam messages by manually giving choice to the users whether message is spam or ham. The technic used by these applications reduces the spread of spam and rumoured messages but this leads to another problem i.e. user needs to read all the message spam then categories it as a spam and ham which is a time-consuming process.

This paper proposes a solution for classifying user's messages into different classes and displaying only those which the user wants. The messages which belong to the class which user does not want is classified as spam messages. This classification process is done with the help of three machine learning algorithm, classifying each and every message of the user.

Literature Review: There exist many papers in which detection is limited to spam and ham within emails and text messages, which perform well for email but not that suitable for social networking applications where different people may have different definition of spam. 
Some papers suggest classification of tweets or messages on the basis of emotions, others suggest comparing and selecting the best suitable machine learning algorithm for text classification for a multiclass system. The algorithm used by most of these papers were Naive Bayes, Support Vector Machine or Random Rain Forest algorithm. Previous work related to spam filtering and text classification are been mentioned below in brief:-

\section{A. Personalized Spam Filtering with Natural Language}

Attributes: Rushdi Shams and Robert E. Mercer proposed a method which reposts the performance of an antispam filter personalized emails named SENTINEL. It uses Natural Language attributes that are related to different components like email readability, grammar, spelling, content words, etc. Categories implemented were Spam and Ham. Also, there are addition to some commonplace attributes, SENTINEL uses attributes related to natural language. The filter used in this paper has been tested with six benchmark datasets in the Enron-Spam collection. the Classifiers which are made by the meta-learning algorithms like ADABOOSTM1 and BAGGING perform equally the best, while a Random Forest (RF) generated classifier performs almost as well. The performance of classifiers using Support Vector Machine (SVM) and Naive Bayes (NB) are not satisfactory for them. The proposed paper shows the comparisons that the performance of SENTINEL surpasses that of a number of state-of-the-art personalized filters proposed in previous studies [Rushdi Shams, 2013].

\section{B. Personalized Spam filtering using Incremental Training} of Support Vector Machine: Gopi Sanghani and Dr. Ketan Kotecha, the paper reposts the personalized spam filter using Support Vector Machine (SVM) with Incremental Training for E-Mails. The filter has two different approaches. First approach, the filter was trained with support vectors incrementally and a collection of incoming mails, keeping the identical set of features. While within the second approach, the feature set is heuristically updated before applying incremental training to SVM. Categories implemented were Legitimate and Spam. The classifier used was Support Vector Machine (SVM). The depth comparison results shows that with conventional SVM batch incremental training, higher classification accuracy and lower false positive rate is achieved [Gopi Sanghani, 2016].

\section{Personalized Spam Filtering with Semi-supervised} Classifier Ensemble: Victor Cheng and C.H. Li, the paper reposts the semi-supervised personalized spam filter based on classifier ensemble classifier that helps the user to label emails for personalizing the spam filter by learning on both generic labelled emails and personalized unlabelled emails. The proposed paper begins learning a SVM model from labelled generic data using multistage classification process. Personalized labelled data for constructing personalized naive Bayes classifiers are generated by feeding unlabelled user's emails to SVM. This paper also exploits some rare word distributions by generating some personalized labelled examples and then fed into semi-supervised classifier. This multi-stage results are integrated with SVMs learned from emails to produce final results. Categories implemented were Spam or not. The classifiers used were Support Vector Machine (SVM), and Naive Bayes (NB). Implemented combination of SVM and NB [Victor Cheng, 2006].

\section{Decentralized and Personalized Spam Filter Based on} Social Computing: Xin Liu, Zhaojun Xin, Leyi Shi, the paper reposts the personalized and collaborative spam filter based on social network to enable users to push spam reports to their social network friends with whom they have similar interest which reflects collaboration and personalization. The spam filtering for emails can be push to social networks. The proposal takes advantage to share user's individual spam knowledge with others via social network, which utilizes wisdom of crowds to resist spam by using push technology. The user can determine whether to push spam reports to his friends with the purpose of taking user's individual interest into consideration. Categories implemented were Interested and Uninterested on basis of user keywords. The classifiers used was Bayesian Filter [Xin Liu, 2014].

Proposed Methodology: In the proposed methodology, the fundamental step is extracting the data from social networks. This data is then pre-processed in which stemming and stop words are removed using Natural Language Processing. This noise-free data is further used for feature extraction which is done by tokenization where the frequency of all the tokens is taken, can be called an array of features. The features and their respective weights are then fed to the classifier to train the model. The trained model is tested on various machine learning algorithms and analysed the accuracy based on predictions of the algorithms.[Hu, 2016] (Fig 1).

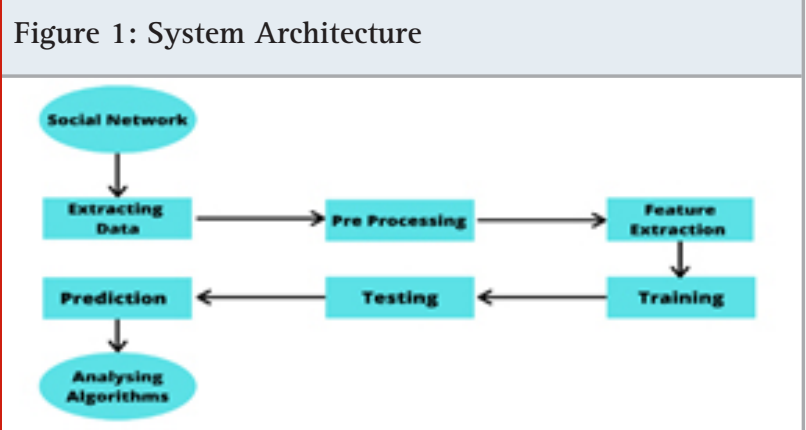

A. Extracting Data: The most important attribute required to train a machine learning algorithm is the dataset. A dataset (Table 1) which had messages from publicity, covid-19, greeting, politics and science and technology classes would solve the purpose, these classes were chosen based on a survey. While searching for dataset on internet there was no dataset which met the expectation for training the model, thus a python code was used to extract messages from some sites. The model would have got a lower accuracy on this dataset as the dataset had noise or unnecessary words, thus filtered the dataset was filtered manually so as to achieve a higher accuracy (and decrease no. of misclassified messages). 
For cleaning the dataset, the following parameters were taken into consideration:

- Removed \#, @ and other irrelevant symbols.

- Removed blank rows.

- Removed URLs via regular expression matching.

- Removed mismatching/incorrect messages.

Table 1. Datasets Category

\begin{tabular}{|l|c|}
\hline $\begin{array}{l}\text { Dataset } \\
\text { Category }\end{array}$ & $\begin{array}{c}\text { Dataset Count } \\
\text { (number of records) }\end{array}$ \\
\hline Covid-19 & 3167 \\
\hline Publicity & 595 \\
\hline Science and Technology & 2439 \\
\hline Greeting & 1012 \\
\hline Politics & 3826 \\
\hline TOTAL & 11039 \\
\hline
\end{tabular}

B. Pre-Processing: The process of converting data to something a computer machine can understand is termed as pre-processing. One of the important forms of pre-processing is to filter out purposeless data in a given dataset. In Natural Language Processing (NLP), this purposeless data, are referred as stopwords. Stopwords are a commonly word used in a sentence (such as 'the', 'is', 'are') that can be filtered from the text to be processed both when indexing for searching or retrieving them as results. These words take extra space memory in a database or take valuable extra time for processing. Therefore, removal of these words is essential for better performance [Daniel Jurafsky, 2019].

NLTK(Natural Language Toolkit) library in Python helps to remove this data. This library of English stopwords contains 153 stopwords. This library also provides PorterStammer Algorithm to reduce all those words which are mapped to the same stem. For instance, play, played, playing all will be viewed as same word. Even punctuations, special symbols and numeric values should be removed from the data as they do not impose a potential impact in the classification process.

C. Feature Extraction: To perform classification, the transformation of features into the numbers is required. Tokenization is a way of separating a piece of text into smaller units called tokens. Tokenization is done using CountVectorizer, which stores the frequency of all the tokens in the form of an array. This can be called as an array of features using which sparse matrix (it consists of multiple zeroes for the words that have not occurred in other documents) is created. Sparse Matrix is further used to adjust the weights of the features to improve the accuracy, which can now be given as input to the classifier [Deepa, 2019].

D. Classification: The first question while solving any machine learning problem is "What machine learning model should I use?", And the answer is always "It depends.", even the most experienced data scientists can’t 378 tell the best performing algorithm before experimenting. That is why the comparison of different multi-class text classification models is done, in order to choose the most accurate one. So the classification models used here are related to supervise machine learning algorithms. For this, the data is categorized into two sets i.e. training set and test set. After training different models with the training dataset, the check on performance for unseen dataset is carried out using test set. In supervised machine learning, various multiclass classification techniques/ methods exists. The one-vs-all technique for multiclass classification is used as shown in (Fig 2).

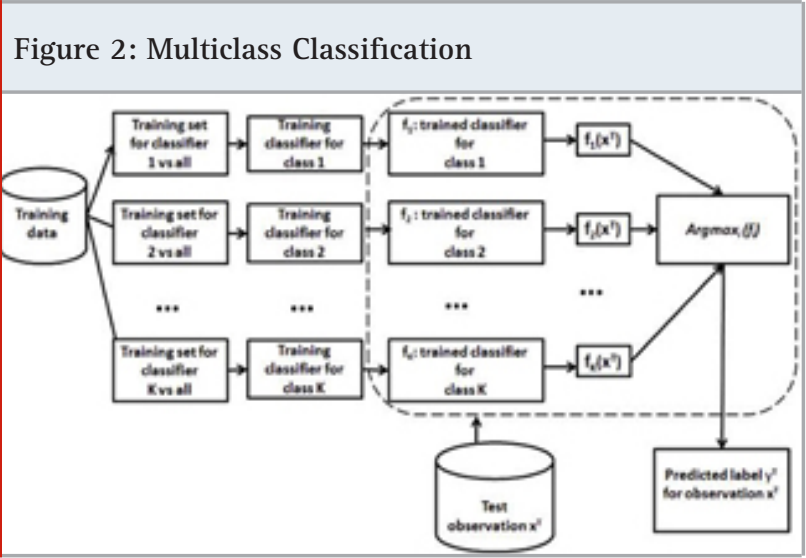

Top 3 most suitable classifiers were identified based on requirements and previous learning. They are:

- Multiclass Support Vector Machine (SVM): SVM is a supervised learning algorithm that is inherently a binary classifier. SVM is based on the principle to learn the maximum marginal hyperplane(MMH) that best segregates the dataset into independent classes. SVM constructs a hyperplane in higher dimensional space to separate different classes that is the relocation of points onto another space where the linear separation of data points is possible. There are several methods to do multiclass classification with SVMs. The method to use depends on the nature of classes whether they are mutually exclusive or not. It is improper to say that classes are independent of each other because classes are hardly statistically independent. The most widely used approach in practice is the "one-versus-all" technique (to build one-versus-rest classifiers) for multiclass classification [Fig. 2]. To generate the binary classifier models for every class individually that separates the rest datapoints of all other classes as one class. [Yi Liu, 2005]

- Multinomial Logistic Regression(LR): Logistic Regression is supervised machine learning algorithm that uses probability for classifying the input. In multinomial logistic regression the target variable ranges over more than two classes. The probability of target variable to belong to a class $\mathrm{c}$ is given by the formula, $\mathrm{c} \in$ $C, p(y=c \mid x)$ [Daniel Jurafsky, 2000].The output of a multinomial logistic regression is a model that explains the relationship between the explanatory variables and the outcome, so that the class of a new input can be 
correctly predicted with the help of the explanatory variables while the outcome is to be predicted.

-Naive Bayes(NB): It is a supervised machine learning algorithm used for classification and is based on Bayes' Theorem. Reason behind the name 'Naive' is the assumption that the presence of a particular feature in a class is independent to the presence of any other feature. This model is basically used for large dataset and is also easy to build. The Naive Bayes algorithm backbone is Bayes Theorem. Here, we calculate the posterior probability (Fig.3) and choose the class with the highest

Table 2. Dataset

\begin{tabular}{|l|c|}
\hline Total Dataset Messages & 11039 \\
\hline Training Data & 10039 \\
\hline Testing Data & 1000 \\
\hline
\end{tabular}

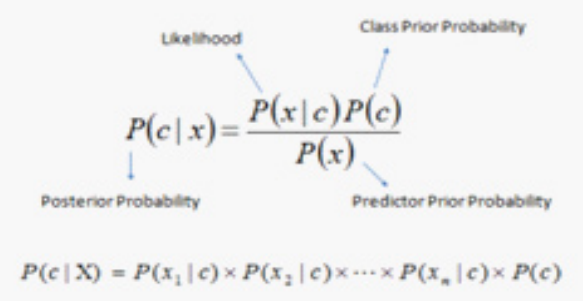

The class with the highest posterior probability is the outcome of prediction. There are 3 main types of Naive Bayes algorithms: -

1. Gaussian Naive Bayes: It is used when features follow a normal distribution.

2. Multinomial Naive Bayes: It is used for discrete counts. Here, this algorithm is implemented.

3. Bernoulli Naive Bayes: This model is useful for binary feature vectors (i.e. 0s and 1s).

Table 3. Results

\begin{tabular}{|l|c|c|c|c|c|c|c|c|}
\hline \multirow{2}{*}{ Classifier } & \multicolumn{4}{|c|}{ Unbalanced Dataset } & \multicolumn{4}{c|}{ Balanced Dataset } \\
\cline { 2 - 9 } & $\begin{array}{c}\text { Training } \\
\text { Time }\end{array}$ & $\begin{array}{c}\text { Testing } \\
\text { Time }\end{array}$ & $\begin{array}{c}\text { Misclassified } \\
\text { Samples }\end{array}$ & Accuracy & $\begin{array}{c}\text { Training } \\
\text { Time }\end{array}$ & $\begin{array}{c}\text { Testing } \\
\text { Time }\end{array}$ & $\begin{array}{c}\text { Misclassified } \\
\text { Samples }\end{array}$ & $\begin{array}{c}\text { Accuracy } \\
\text { NB }\end{array}$ \\
\hline LR & 1.026578 & 0.000838 & 43 & 0.9586 & 0.042360 & 0.000998 & 55 & 0.9471 \\
\hline SVM & 238.375338 & 24.619462 & 78 & 0.924927 & 828.471336 & 48.092569 & 69 & 0.933589 \\
\hline
\end{tabular}

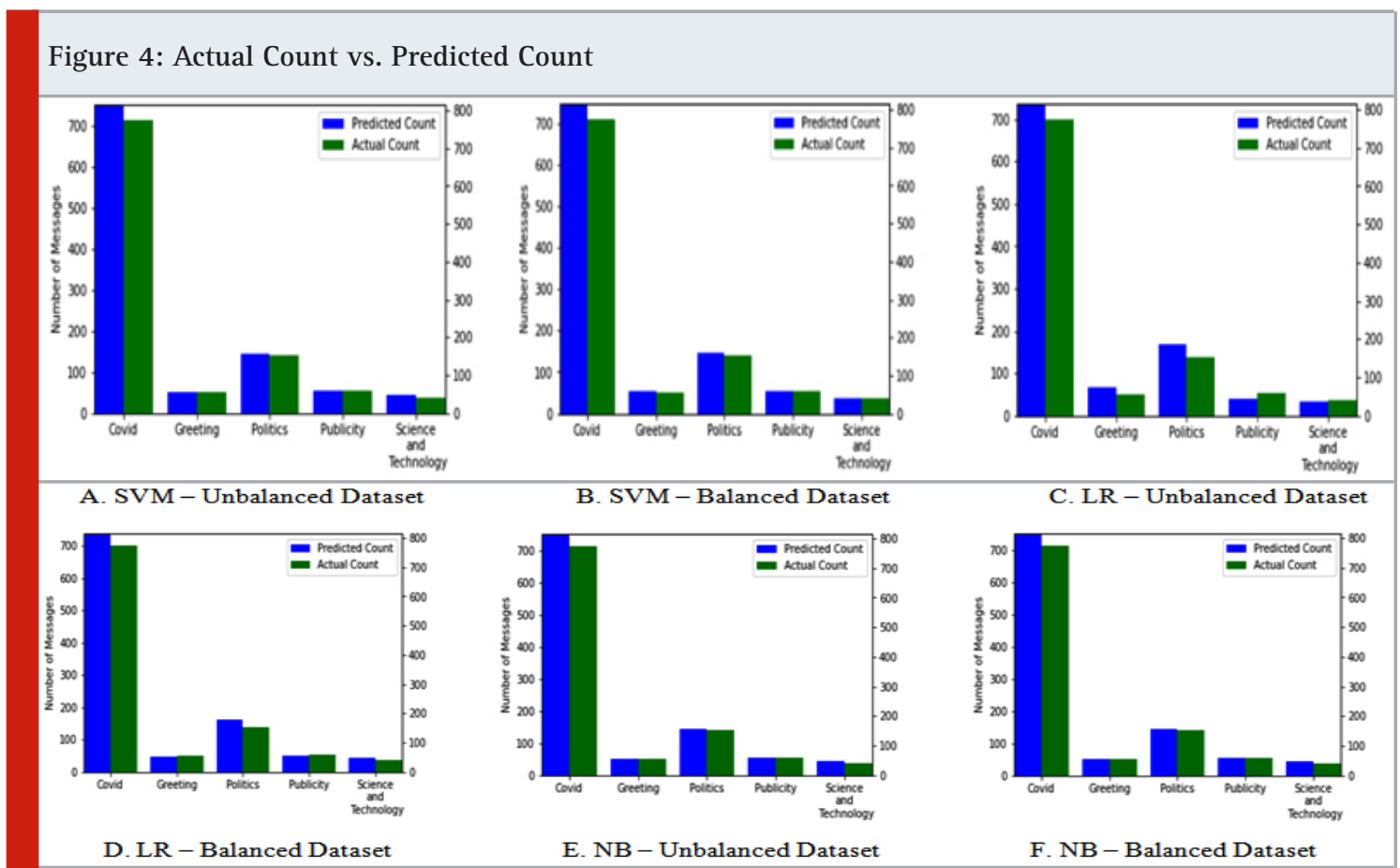

\section{RESULTS}

In (Fig 4), the actual and predicted count of samples of different classes on SVM, Logistic Regression, and
Naïve Bayes is shown. A balanced and unbalanced dataset is taken into consideration for better analysis across different algorithms. Table- 3 describes various important parameters of each algorithm on unbalanced 
and balanced data. It shows total training time, Testing time, accuracy, and the number of misclassified samples for all the three algorithms. First, the model is trained using an unbalanced dataset and achieved high accuracy of 96\% for the Naïve Bayes algorithm, and also the response time was comparatively very fast. There was a huge difference in the number of samples per class. Therefore, balancing the dataset was attempted which contains an equal number of samples of all the classes in the dataset, where Logistic Regression showed 95\% accuracy and also better response time. Balancing dataset failed to show overall progress but in case of SVM and Logistic Regression, there was a considerable increase in accuracy respectively.

First, the model was trained using unbalanced dataset and achieved high accuracy of 96\% for Naïve Bayes algorithm and also the response time was comparatively very fast. There was a huge difference in the number of samples per classes. Therefore, when balancing was done i.e. that contains equal number of samples of all the classes in the dataset, where Logistic Regression showed 95\% of accuracy and also better response time. Balancing dataset failed to show overall progress but in case of SVM and Logistic Regression there was a considerable increase in accuracy respectively.

\section{CONCLUSION}

Here, the comparative study of three supervised machine learning classifiers (Naive Bayes, Logistic Regression, SVM) for personalized spam filter of social network is shown (Fig 4). Through results, the conclusion was that, Naive Bayes is the best performing algorithm for multiclass text classification with large data set as studied earlier. Also the time taken for training and testing was very less in Naive Bayes and Logistic Regression as compared to SVM. The accuracy of SVM and Logistic Regression increased on balanced dataset as compared to unbalanced dataset while it decreased in the case of Naive Bayes, but still was best among all. The future goal is to check the news for true information using web scrapping. This will help to combat the rapid spread of fake news on social network. Another future goal is to increase the dataset automatically from the user and hence producing more accurate results with more dataset.

\section{REFERENCES}

Ashwini Rahangdale, Avinash Agrawal, "INFORMATION EXTRACTION USING DISCOURSE ANALYSIS FROM NEWSWIRES", International Journal of Information Technology Convergence and Services (IJITCS) Vol.4, No.3, June 2014, DOI:10.5121/ijitcs.2014.4302, ISSN : 2231 - 153X (Online) ; 2231 - 1939 (Print), pp.21
Daniel Jurafsky \&t James H. Martin, Speech and Language Processing, 2019.

Daniel Jurafsky and James H. Martin .Speech and Language Processing: An Introduction to NaturalLanguage Processing, Computational Linguistics, and Speech Recognition. Prentice Hall PTR, USA, 2nd edition, 2000

Deepa, D., Raaji, and Tamilarasi A. "'Sentiment Analysis using Feature Extraction and Dictionary-Based Approaches" Third International Conference on I-SMAC (IoT in Social, Mobile, Analytics and Cloud) (I-SMAC) , 2019.

Duan, K.-B. and Keerthi, S. S.,"Which Is the Best Multiclass SVM Method? An Empirical Study" International Workshop on Multiple Classifier System, 2005.

Gopi Sanghani and Dr. Ketan Kotecha, "Personalized Spam filtering using Incremental Training of Support Vector Machine”, 2016 International Conference on Computing, Analytics and Security Trends (CAST), College of Engineering Pune, India. Dec 19-21, 2016.

Hira, Z. M., and Gillies, D. F. "A Review of Feature Selection and Feature Extraction Methods Applied on Microarray Data” Advances in Bioinformatics, 2015.

$\mathrm{Hu}$, W., Du, J., and Xing, Y. , "Spam filtering by semantics-based text classification” , Eighth International Conference on Advanced Computational Intelligence (ICACI), 2016 .

Rushdi Shams and Robert E. Mercer, "Personalized Spam Filtering with Natural Language Attributes”, 2013 12th International Conference on Machine Learning and Applications.

Snehal Dixit, Avinash J Agrawal, "Survey on Review Spam Detection”, International Journal of Computer \& Communication Technology, Volume 4, Issue 2, 2013, ISSN: 0975-7449, 68-72

Victor Cheng and C.H. Li, "Personalized Spam Filtering with Semi-supervised Classifier Ensemble”, Proceedings of the 2006 IEEE/WIC/ACM International Conference on Web Intelligence (WI 2006 Main Conference Proceedings) (WI'06).

Xin Liu, Zhaojun Xin, Leyi Shi, and Yao Wang, "A Decentralized and Personalized Spam Filter Based on Social Computing”, 2014 International Wireless Communications and Mobile Computing Conference (IWCMC).

Yi Liu and Yuan F. Zheng, One-Against-All Multi-Class SVM Classification Using Reliability Measures, IEEE International Joint Conference on Neural Networks, 2005. 